\title{
The pluripotency transcription factor network at work in reprogramming
}

\author{
Hitoshi Niwa1,2
}

1Laboratory for Pluripotent Stem Cell Studies

RIKEN Center for Developmental Biology

2-2-3 Minatojima-minamimachi, Chuo-ko, Kobe 650-0047

Japan

2CREST, Sanbancho, Chiyodaku, Tokyo, 1020075, Japan

niwa@cdb.riken.jp

\begin{abstract}
Pluripotency-associated transcription factors possess a pivotal role to maintain pluripotency in pluripotent stem cells as well as to induce pluripotency in somatic cells. They direct specific pattern of gene expression from the genome by co-operating with the genetic and epigenetic mechanisms. Recent findings revealed that these mechanisms possess unique features in pluripotent stem cells, which is different from that in somatic cells either qualitatively and quantitatively. To reprogram somatic cells, pluripotency-associated transcription factors should modulate the co-operating maschneries to establish the optimal environment for their function to maintain pluripotency-associated transcription factor network.
\end{abstract}

\section{Introduction}

Pluripotency is defined as an ability of a cell to differentiate into all somatic cell types. It is observed in limited numbers of cells in pre- and early-post implantation embryos in developmental context. Once cultured in vitro, these cells derive pluripotent stem cells with continuous self-renewal. Somatic cells including stem cells of post-natal mice never give rise to pluripotent stem cells without reprogramming. Reprogramming is an event that resets the genetic and epigenetic information in a genome-wide manner. Reprogramming of somatic cell nuclei to totipotent state is achieved by the nuclear transfer technique[1], whereas reprogramming of somatic cells into pluripotent stem cells is induced by the over-expression of pluripotency-associated transcription 
factors[2]. It has been reported that several molecules are involved in the reprogramming event, but it is still unclear how the pluripotency-associated transcription factor network is finally established to maintain the pluripotent state induced by reprogramming. Here the recent findings were reviewed to understand the reprogramming event from the point of view of the transcription factor network transition.

\section{A. Transcription factor network in pluripotent stem cells}

Mouse embryonic stem (ES) cells continue self-renewal in the presence of leukemia inhibitory factor (LIF). LIF signal integrates into ES cells and activates three different intracellular signal transduction pathways, which target the expressions and activities of multiple pluripotency-associated transcription factors such as Klf4, Tbx3 and $T f c p 2 l 1[3,4]$. Wnt signal cooperates with LIF signal to promote the maintenance of pluripotency via transcriptional activation of the transcription factor Esrrb [5]. These transcription factors form a network in which they regulate each other and process the signal integration to direct self-renewal (or to block differentiation which is a default) by maintaining the expressions of the core transcription factors that consist of Oct3/4 and Sox2 (Figure 1). The core transcription factors compose auto-regulatory loops to maintain their own expressions and regulate the components of the pluripotency-associated transcription factor network in a signal integration-dependent manner[6]. Moreover, transcription factor network possesses a positive feedback to amplify LIF signal integration via transcriptional repression of Socs3, a negative regulator of Stat3 activation, by Nanog[7]. The essence of the function of the transcription factor network is the maintenance of pluripotency.

\section{Transcriptional activation by transcription factors}

Mediating the functions of pluripotency-associated transcription factors involves many parameters that define their abilities to activate or repress the target gene expression. This includes the interactions among the transcription factors, transcriptional co-factors and general transcription factors.

\section{Interaction of the transcription factors}

It has been reported that Oct $3 / 4$ and Sox 2 form a heterodimer to activate the 
transcription of the target genes including their own. The direct interactions of transcription factors define their specific functions[6]. For example, Sox2 is expressed in a variety of cell types during development including trophoblast stem (TS) cells[8]. In TS cells, Sox 2 functions to mediate the mitogen-activated protein kinase (MAPK) signal to maintain self-renewal[9] although it is known that the MAPK signal negatively regulates pluripotency in ES cells[10]. Moreover, Sox 2 regulates a set of target genes that are different from ES cells, including TS cell-specific transcription factors, and the differential mode of function is partly due to the interaction of Sox 2 with Tfap2c in TS cells[9]. Therefore, a proper combination of the transcription factors is required to direct their specific function, and the combination of Sox 2 with Oct $3 / 4$ confers the function to regulate the pluripotency-associated genes. In ES cells, Klf4 is known to act as a partner of Oct3/4 and Sox 2 to activate the transcription of particular target genes such as Lefty1[11], which might partly explain the requirement of Klf4 as a reprogramming factor. However, it is still unclear by how many transcription factors the complete function is determined, since the addition of transcription factors to the Yamanaka factors (Oct3/4, Sox2, Klf4 and Myc) still showed weak synergistic effect to promote reprogramming efficiency[12].

Recently, the concept of 'super-enhancer' has been established[13,14]. On the tissue-specific enhancers, multiple $(\sim 8)$ transcription factors form a large complex to direct transcriptional activation. This concept supports the idea that the 4 transcription factors are NOT sufficient to direct full activation of the target genes.

\section{Mediator complex}

To direct transcriptional activation, multiple transcription factors bind to the enhancer located at the distal site of the promoter. The binding of these transcription factors is transduced by the mediator complex to recruit general transcription factors to the promoter, which promote the recruitment of RNA polymerase II complex (polII)[15] (Figure 2). Mediator complex consists of $\sim 30$ components and is regarded as a general transcriptional machinery shared by all types of cells. However, it was reported that the knockout of the components show cell-type-specific defect and that the tissue-specific transcription factors interact with different components of the mediator complex, and the expression levels of the components could vary in different cell types[15]. Therefore, it is possible that proper combinations of the transcription factors and the mediator 
components are required for proper function of the transcription factors to establish and maintain the transcription factor network, and the functions of the ectopically-expressed transcription factors could be blocked by the endogenous transcription factors by competing for the common interaction partner of the mediator complex. Moreover, it has been reported that the mediator complex interacts with cohesin and CTCF to shape $3 \mathrm{D}$ organization of the genome[16,17], suggesting the importance of the interaction to the mediator complex beyond the direct regulation of transcription.

\section{General transcription factors}

The general transcription factors bind to the promoter to recruit polII and are regarded to have ubiquitous functions in all cell types[18] (Figure 2). TFIID is a basal transcription factor complex containing TATA-binding protein (TBP) and 13 TBP-associated factors (TAFs) [18]. Pijnappel et al demonstrated that TBP and many TAFs are expressed at higher levels in ES cells than in somatic cells and their rather high expression levels are required for the maintenance of pluripotency as well as the reprogramming of somatic cells[19]. Interestingly, some of the TFIID subunits like TAF4 are directly regulated by Oct3/4, indicating that their high expression levels are partly conferred by the pluripotency-associated transcription factor network thus forming a positive feedback loop for the maintenance of pluripotency[19]. Since there are cell-type-specific paralogs of TFIID subunits[18], the composition of TFIID could also affect the transcriptional activity of the ectopically-expressed pluripotency-associated transcription factors in somatic cells.

\section{Remodeling complex}

Nuclear remodeling is an essential part of proper transcriptional activation since its function alters the accessibility to the genomic DNA for both tissue-specific and general transcription factors as well as polII (Figure 2). It has been known that ES cells possess a special type of the SWI/SNF complex, esBAF[20,21]. esBAF contains specific components that are required for the interaction to the pluripotency-associated transcription factors such as Oct3/4 and Sox2[20]. Therefore, the functions of Oct3/4 and Sox 2 in somatic cells without esBAF should be limited compared to those in ES cells. The NuRD complex is known as a negative regulator of pluripotency by 
interfering with the expression of the transcription factor genes[22]. It was also reported that Mbd3, a component of the NuRD complex, is a potent limiter of the reprogramming event[23]. Therefore, the NuRD complex may possess specific functions in pluripotent stem cells that may affect the functions of the ectopically-expressed pluripotency-associated transcription factors in somatic cells. In addition, the role of the INO80 complex for efficient reprogramming, ES self-renewal and blastocyst formation has also been reported[24].

\section{THO complex/RNA export}

The THO complex coordinates mRNA transcription and export from the nuclei (Figure 2). Two components of the THO complex, Thoc2 and Thoc5, are highly expressed in ES cells. Their knock-down resulted in morphological changes with repression of pluripotency-associated transcription factors in ES cells. Their expression increased quickly during reprogramming, which led to efficient reprogramming of the somatic cells[25]. Therefore, the coupling of THO complex confers proper functions to the Yamanaka factors.

\section{Modulation of the transcription factor activities}

The binding of transcription factors to the target sites depends on its accessibility. Chromatin conformation is regulated by multiple modes of the epigenetic regulation, and in some of them the abilities to ensure the functions of pluripotency-associated transcription factors has been confirmed.

\section{Histone modifiers}

Histone modifications modulate the activities of the transcription factors by regulating the accessibility to the chromatin. Direct interaction of the pluripotency-associated transcription factors with the epigenetic modifiers were reported: Oct3/4 with Wdr5, a component of trithorax complex that mediates H3K4 tri-methylation[26], and Nanog with Mof, a histone acetyltransferase for H4K16[27], which are functional interactions to maintain self-renewal in ES cells.

\section{Non-coding RNA}

Long non-coding (lnc) RNAs are emerging factors involved in the 
maintenance of ES cell-specific gene expression. Guttmann et al reported that a set of lncRNAs is specifically expressed in ES cells and some of them are involved in the maintenance of pluripotency in ES cells[28]. Interestingly, the pluripotency-associated transcription factors directly regulate the expression of a part of them, and their function may be exerted via the interaction with the epigenetic regulators[27]. Moreover, recent

report demonstrated that Wdr5 binds to lncRNAs to exert its function for the maintenance of pluripotency in ES cells[29].

It was known that ES cells express retrotransposon-derived transcripts and long-terminal repeat (LTR) sequences are enriched in stem-cell specific lncRNAs[30], and LTRs serve as binding sites for Oct3/4 and Nanog[31]. Recently, Fort et al identified a class of ES cell-specific transcripts originate in the LTR elements, and showed that some of their expressions are associated with the maintenance of pluripotency via the interaction with distal regulatory elements in ES cells[32]. These evidences suggest that lncRNAs are involved in the induction and maintenance of the pluripotency-associated transcription factor network.

\section{Functions of transcription factors in reprogramming}

To drive the reprogramming process of somatic cells into pluripotent stem cells, the Yamanaka factors are expressed ectopically in somatic cells. It is easy to speculate that these transcription factors are unable to work as in ES cells because of the differences in the points mentioned above: First, the number of the pluripotency-associated transcription factors may be too low to firmly exert their functions via the interaction of each others. In addition, lack of the partner molecules of the mediator complex and the remodeling complex, low expression of the general transcription factors and the epigenetic regulators, and the differential expression of non-coding RNAs could also have negative effects. The Yamanaka factors should overcome these barriers to establish pluripotency, which may occur in a stochastic manner along the time course of reprogramming.

How do the transcription factors work at the initial phase of reprogramming in somatic cells? Soufi et al analyzed the genome-wide binding patterns of of OCT3/4, SOX2, KLF4 and MYC at 48 hours after their induction in human fibroblasts and compared them to those in human ES cells[33]. As the result, they found that OCT3/4, SOX2, KLF4 and MYC preferencially bind together to the distal regions of the 
promoters of the transcriptionally-inactive genes. This is in contrast to the binding of these transcription factors in human ES cells; co-occupation by these 4 factors is rare and MYC and KLF4 preferentially bind to the promoter regions of the transcriptionally-active genes. Interestingly, OCT3/4, SOX2 and KLF4 act as pioneer factors that bind to the targets without pre-existing factors whereas MYC enhances their binding without the function as a pioneer factor. However, large chromatin domains with H3K9me3 prevent the binding of OCT3/4, SOX2 and KLF4 as found in the genomic region including $N A N O G$. This is consistent with the finding that knock-down of $S U V 39 H 1$ facilitate reprogramming (see below).

\section{Obstacles for the function of transcription factors in reprogramming}

To progress reprogramming, the Yamanaka factors should activate multiple genes to establish a proper activity of the pluripotency-asociated transcription factor network that works in a signal-dependent manner. However, there are many obstacles that specifically affect the establishment, but not the maintenance, of pluripotency. This includes both the genetic and epigenetic mechanisms.

Competition by tissue-specific transcription factors

The phenotypes of the somatic cells are determined by the activities of the cell-type-specific transcription factor network. The activity may facilitate or interfere with the function of the Yamanaka factors. It was reported that the terminally-differentiated B lymphocytes were hardly reprogrammed by Yamanaka factors but the knockdown of Pax5 enhanced the efficiency[34]. Recently, Hikichi et al reported that a particular set of tissue-specific transcription factors compete with the reprogramming by the Yamanaka factors although the precise molecular mechanism is still unknown[35].

\section{DNA methylation}

DNA methylation is a major epigenetic modification coupled with heritable heterochromatin. The Ten eleven translocation (Tet) family encoding methylcytosine hydroxylase are implicated in DNA demethylation. Tet family consists of three members (Tet1, 2 and 3) and the knockout of all of them in mouse embryonic fibroblasts (MEFs) abolish their competence for reprogramming[36] although the triple 
knockout ES cells continue self-renewal[37]. Interestingly, the competency of MEFs lacking all Tet genes for reprogramming is restored by the addition of micro RNAs to the Yamanaka factors[36]. It was reported that Thymine DNA glycosylase (TDG) and Aid also involve in DNA demethylation. Loss of TDG blocks reprogramming[36], but the Aid-deficient MEFs are capable of normal reprogramming[38]. Therefore, in the reprogramming process, the Tet-TDG pathway is important to facilitate DNA demethylation although they are dispensable for the maintenance of pluripotency.

\section{Histone modifiers}

Loss-of-function screening of chromatin-modifying enzymes for their abilities to modulate reprogramming efficiency of human somatic cells revealed that inhibition of PRC1 (BMI1, RING1) and PRC2 components (EZH2, EED, SUZ12) significantly decreased reprogramming efficiency whereas inhibition of YY1, SUV39H1 and DOTL1 enhanced reprogramming[39].

Dotll encodes H3K79 methyltransferase and it was reported that Dotll-null ES cells show global loss of H3K79 methylation and reduced levels of heterochromatic markers such as H3K9 di-methylation and H4K20 tri-methylation[40]. However, they can proliferate normally with keeping the morphological feature although showing defect in cell-cycle after differentiation[41], suggesting its specific function in the induction, but not in the maintenance, of pluripotency.

\section{Conclusions and future perspectives}

The cell-type-specific transcription factors act as a primary determinant of cellular phenotypes. However, to achieve their proper functions, many transcriptional co-factors and epigenetic regulators should work together in correct combinations. This is one of the reasons why the reprogramming or trans-differentiation require multiple transcription factors and are achieved at low efficiency. It is in contrast to the differentiation events along the developmental time course that can be triggered in vitro with a single transcription factor at high efficiency, probably due to the cellular context that is compatible to allow proper function of the ectopically-expressed transcription factor[42,43].

There are possible obstacles to the reprogramming events in addition to those shown above. Nuclear import of the transcription factors is essential for them to 
function. Importin $\alpha$ subtypes determine nuclear localization of transcription factors. It was reported that Importin $\alpha 2$ inhibits the nuclear import of neuronal transcription factors Oct6 and Brn2 in ES cells and that the C-terminal region of Importin $\alpha$ proteins determines the binding and import of the partner proteins[44]. Therefore, the compatibility of the ectopically-expressed transcription factors to the class of Importin $\alpha$ subtypes expressed in the host cells could restrict the function. Post-translational modifications may also regulate the transcription factors ectopically expressed in somatic cells.

Modulation of the parameters indicated in this review can enhance the reprogramming by the Yamanaka factors. One important aspect to modulate the parameters is how to make the closed chromatin structure open as found in ES cells[6]. One interesting example is the effect of histone variants on reprogramming. The histone variants TH2A and TH2B are expressed in oocytes but not in ES cells[45]. Co-expression of them with Yamanaka factors and histone chaperone enhances the efficiency of reprogramming with kinetic acceleration. Interestingly, the nucleosome containing $\mathrm{TH} 2 \mathrm{~A}$ and $\mathrm{TH} 2 \mathrm{~B}$ take a more open structure compared to the nucleosome containing $\mathrm{H} 2 \mathrm{~A}$ and $\mathrm{H} 2 \mathrm{~B}[45]$. Therefore, an artificial modulation of the chromatin structure that makes it open, in combination with the transcription factors, will be a central dogma of the efficient reprogramming, which might have been achieved in functional ablation of Mbd3 that directed the highest efficiency of reprograming to date[23] althugh there are contrivertial reports[39,46]. The mechanisms conferring the link between the pluripotency-associated transcription factors and the open chromatin structure should be revealed to understand the reprogramming event.

\section{Acknowledgements}

We apologize to colleagues, whose work could not be cited because of space limitations and was covered by reviews instead. We thank Yoko Futatsugi-Nakai for critical reading of manuscript. This work is supported by CREST program of the Japan Science and technology Agency (JST) and RIKEN grant.

\section{References and recommended reading}

\section{Gurdon JB, Elsdale TR, Fischberg M: Sexually mature individuals of Xenopus}


laevis from the transplantation of single somatic nuclei. Nature 1958, 182:64-65.

2. Takahashi K, Yamanaka S: Induction of pluripotent stem cells from mouse embryonic and adult fibroblast cultures by defined factors. Cell 2006, 126:663-676.

3. Niwa H, Ogawa K, Shimosato D, Adachi K: A parallel circuit of LIF signalling pathways maintains pluripotency of mouse ES cells. Nature 2009, 460:118-122.

4. Martello G, Bertone P, Smith A: Identification of the missing pluripotency mediator downstream of leukaemia inhibitory factor. Embo $J 2013$, 32:2561-2574.

5. Martello G, Sugimoto T, Diamanti E, Joshi A, Hannah R, Ohtsuka S, Gottgens B, Niwa H, Smith A: Esrrb is a pivotal target of the Gsk3/Tcf3 axis regulating embryonic stem cell self-renewal. Cell Stem Cell 2012, 11:491-504.

6. Niwa H: How is pluripotency determined and maintained? Development 2007, 134:635-646.

7. Stuart HT, van Oosten AL, Radzisheuskaya A, Martello G, Miller A, Dietmann S, Nichols J, Silva J: NANOG amplifies STAT3 activation and they synergistically induce the naïve pluripotent program. Curr Biol 2014, 24:340-346.

8. Avilion AA, Nicolis SK, Pevny LH, Perez L, Vivian N, Lovell-Badge R: Multipotent cell lineages in early mouse development depend on SOX2 function. Genes Dev 2003, 17:126-140.

9. Adachi K, Nikaido I, Ohta H, Ohtsuka S, Ura H, Kadota M, Wakayama T, Ueda HR, Niwa H: Context-dependent wiring of Sox 2 regulatory networks for self-renewal of embryonic and trophoblast stem cells. Mol Cell 2013, 52:380-392.

10. Ying QL, Wray J, Nichols J, Batlle-Morera L, Doble B, Woodgett J, Cohen P, Smith A: The ground state of embryonic stem cell self-renewal. Nature 2008, 453:519-523.

11. Nakatake Y, Fukui N, Iwamatsu Y, Masui S, Takahashi K, Yagi R, Yagi K, Miyazaki J, Matoba R, Ko MS, et al.: Klf4 cooperates with Oct3/4 and Sox 2 to activate the Lefty1 core promoter in embryonic stem cells. Mol Cell Biol 2006, 
26:7772-7782.

12. Maekawa M, Yamaguchi K, Nakamura T, Shibukawa R, Kodanaka I, Ichisaka T, Kawamura Y, Mochizuki H, Goshima N, Yamanaka S: Direct reprogramming of somatic cells is promoted by maternal transcription factor Glis1. Nature 2011, 474:225-229.

13. Hnisz D, Abraham BJ, Lee TI, Lau A, Saint-Andre V, Sigova AA, Hoke HA, Young RA: Super-enhancers in the control of cell identity and disease. Cell 2013, 155:934-947.

14. Whyte WA, Orlando DA, Hnisz D, Abraham BJ, Lin CY, Kagey MH, Rahl PB, Lee TI, Young RA: Master transcription factors and mediator establish super-enhancers at key cell identity genes. Cell 2013, 153:307-319.

** This is the first study that proposed the concept of 'super-enhancer' where multiple cell-type-specific transcription factors accumulate to direct cell-type specificity.

15. Yin JW, Wang G: The Mediator complex: a master coordinator of transcription and cell lineage development. Development 2014, 141:977-987.

16. Kagey MH, Newman JJ, Bilodeau S, Zhan Y, Orlando DA, van Berkum NL, Ebmeier CC, Goossens J, Rahl PB, Levine SS, et al.: Mediator and cohesin connect gene expression and chromatin architecture. Nature 2010, 467:430-435.

** This is the first study that demonstarted the role of the mediator complex for both transcriptional activation from the distal enhancers and chromatin architecture via interaction with cohesin.

17. Phillips-Cremins JE, Sauria ME, Sanyal A, Gerasimova TI, Lajoie BR, Bell JS, Ong CT, Hookway TA, Guo C, Sun Y, et al.: Architectural protein subclasses shape 3D organization of genomes during lineage commitment. Cell 2013, 153:1281-1295.

18. Muller F, Zaucker A, Tora L: Developmental regulation of transcription initiation: more than just changing the actors. Curr Opin Genet Dev 2010, 20:533-540.

19. Pijnappel WW, Esch D, Baltissen MP, Wu G, Mischerikow N, Bergsma AJ, van der Wal E, Han DW, Bruch H, Moritz S, et al.: A central role for TFIID in the pluripotent transcription circuitry. Nature 2013, 495:516-519. 
** This study indicates the significance of the high level expression of the general transcription factor TFIID for proper activation of the pluripotency-associated genes.

20. Ho L, Ronan JL, Wu J, Staahl BT, Chen L, Kuo A, Lessard J, Nesvizhskii AI, Ranish J, Crabtree GR: An embryonic stem cell chromatin remodeling complex, esBAF, is essential for embryonic stem cell self-renewal and pluripotency. Proc Natl Acad Sci U S A 2009, 106:5181-5186.

21. Ho L, Jothi R, Ronan JL, Cui K, Zhao K, Crabtree GR: An embryonic stem cell chromatin remodeling complex, esBAF, is an essential component of the core pluripotency transcriptional network. Proc Natl Acad Sci U S A 2009, 106:5187-5191.

22. Reynolds N, Latos P, Hynes-Allen A, Loos R, Leaford D, O'Shaughnessy A, Mosaku O, Signolet J, Brennecke P, Kalkan T, et al.: NuRD suppresses pluripotency gene expression to promote transcriptional heterogeneity and lineage commitment. Cell Stem Cell 2012, 10:583-594.

23. Rais Y, Zviran A, Geula S, Gafni O, Chomsky E, Viukov S, Mansour AA, Caspi I, Krupalnik V, Zerbib M, et al.: Deterministic direct reprogramming of somatic cells to pluripotency. Nature 2013, 502:65-70.

* This study identifed Mbd3, a component of NurD complex, as a key factor to antagonize the reprogramming by Yamanaka factors.

24. Wang L, Du Y, Ward JM, Shimbo T, Lackford B, Zheng X, Miao YL, Zhou B, Han L, Fargo DC, et al.: INO80 Facilitates Pluripotency Gene Activation in Embryonic Stem Cell Self-Renewal, Reprogramming, and Blastocyst Development. Cell Stem Cell 2014, 14:575-591.

25. Wang L, Miao YL, Zheng X, Lackford B, Zhou B, Han L, Yao C, Ward JM, Burkholder A, Lipchina I, et al.: The THO complex regulates pluripotency gene mRNA export and controls embryonic stem cell self-renewal and somatic cell reprogramming. Cell Stem Cell 2013, 13:676-690.

26. Ang YS, Tsai SY, Lee DF, Monk J, Su J, Ratnakumar K, Ding J, Ge Y, Darr H, Chang $B$, et al.: Wdr5 mediates self-renewal and reprogramming via the embryonic stem cell core transcriptional network. Cell 2011, 145:183-197.

27. Li X, Li L, Pandey R, Byun JS, Gardner K, Qin Z, Dou Y: The histone acetyltransferase MOF is a key regulator of the embryonic stem cell core 
transcriptional network. Cell Stem Cell 2012, 11:163-178.

28. Guttman M, Donaghey J, Carey BW, Garber M, Grenier JK, Munson G, Young G, Lucas AB, Ach R, Bruhn L, et al.: lincRNAs act in the circuitry controlling pluripotency and differentiation. Nature 2011, 477:295-300.

* This study identifed several pluripotency-associated lncRNAs and revealed their function in cooperation with epigenetic factors.

29. Yang YW, Flynn RA, Chen Y, Qu K, Wan B, Wang KC, Lei M, Chang HY:

Essential role of IncRNA binding for WDR5 maintenance of active chromatin and embryonic stem cell pluripotency. Elife 2014, 3:e02046.

30. Kelley D, Rinn J: Transposable elements reveal a stem cell-specific class of long noncoding RNAs. Genome Biol 2012, 13:R107.

31. Kunarso G, Chia NY, Jeyakani J, Hwang C, Lu X, Chan YS, Ng HH, Bourque G: Transposable elements have rewired the core regulatory network of human embryonic stem cells. Nat Genet 2010, 42:631-634.

32. Fort A, Hashimoto K, Yamada D, Salimullah M, Keya CA, Saxena A, Bonetti A, Voineagu I, Bertin N, Kratz A, et al.: Deep transcriptome profiling of mammalian stem cells supports a regulatory role for retrotransposons in pluripotency maintenance. Nat Genet 2014.

* This study identified retrotransposon-associated lncRNAs specifically expressed in pluripotent stem cells and revealed their functions to maintain pluripotency.

33. Soufi A, Donahue G, Zaret KS: Facilitators and impediments of the pluripotency reprogramming factors' initial engagement with the genome. Cell 2012, 151:994-1004.

** This study showed the initial phase of the reporgramming following the binding of the Yamanaka factors on the genome.

34. Hanna J, Markoulaki S, Schorderet P, Carey BW, Beard C, Wernig M, Creyghton MP, Steine EJ, Cassady JP, Foreman R, et al.: Direct reprogramming of terminally differentiated mature B lymphocytes to pluripotency. Cell 2008, 133:250-264.

35. Hikichi T, Matoba R, Ikeda T, Watanabe A, Yamamoto T, Yoshitake S, Tamura-Nakano M, Kimura T, Kamon M, Shimura M, et al.: Transcription factors interfering with dedifferentiation induce cell type-specific transcriptional profiles. Proc Natl Acad Sci U S A 2013, 110:6412-6417. 
36. Hu X, Zhang L, Mao SQ, Li Z, Chen J, Zhang RR, Wu HP, Gao J, Guo F, Liu W, et al.: Tet and TDG Mediate DNA Demethylation Essential for Mesenchymal-to-Epithelial Transition in Somatic Cell Reprogramming. Cell Stem Cell 2014, 14:512-522.

37. Dawlaty MM, Breiling A, Le T, Barrasa MI, Raddatz G, Gao Q, Powell BE, Cheng AW, Faull KF, Lyko F, et al.: Loss of tet enzymes compromises proper differentiation of embryonic stem cells. Dev Cell 2014, 29:102-111.

38. Shimamoto R, Amano N, Ichisaka T, Watanabe A, Yamanaka S, Okita K: Generation and characterization of induced pluripotent stem cells from aid-deficient mice. PLoS One 2014, 9:e94735.

39. Onder TT, Kara N, Cherry A, Sinha AU, Zhu N, Bernt KM, Cahan P, Marcarci BO, Unternaehrer J, Gupta PB, et al.: Chromatin-modifying enzymes as modulators of reprogramming. Nature 2012, 483:598-602.

40. Jones B, Su H, Bhat A, Lei H, Bajko J, Hevi S, Baltus GA, Kadam S, Zhai H, Valdez R, et al.: The histone H3K79 methyltransferase Dot1L is essential for mammalian development and heterochromatin structure. PLoS Genet 2008, 4:e1000190.

41. Barry ER, Krueger W, Jakuba CM, Veilleux E, Ambrosi DJ, Nelson CE, Rasmussen TP: ES cell cycle progression and differentiation require the action of the histone methyltransferase Dot1L. Stem Cells 2009, 27:1538-1547.

42. Fujikura J, Yamato E, Yonemura S, Hosoda K, Masui S, Nakao K, Miyazaki Ji J, Niwa H: Differentiation of embryonic stem cells is induced by GATA factors. Genes Dev 2002, 16:784-789.

43. Niwa H, Toyooka Y, Shimosato D, Strumpf D, Takahashi K, Yagi R, Rossant J: Interaction between Oct $3 / 4$ and $\mathrm{Cdx} 2$ determines trophectoderm differentiation. Cell 2005, 123:917-929.

44. Yasuhara N, Yamagishi R, Arai Y, Mehmood R, Kimoto C, Fujita T, Touma K, Kaneko A, Kamikawa Y, Moriyama T, et al.: Importin alpha subtypes determine differential transcription factor localization in embryonic stem cells maintenance. Dev Cell 2013, 26:123-135.

45. Shinagawa T, Takagi T, Tsukamoto D, Tomaru C, Huynh LM, Sivaraman P, Kumarevel T, Inoue K, Nakato R, Katou Y, et al.: Histone variants enriched in oocytes enhance reprogramming to induced pluripotent stem cells. Cell Stem 
Cell 2014, 14:217-227.

46. Dos Santos RL, Tosti L, Radzisheuskaya A, Caballero IM, Kaji K, Hendrich B,

Silva, JC: MBD3/NuRD facilitates induction of pluripotency in a

context-dependent manner. Cell Stem Cell 2014, 15:102-110.

\section{Figure legends}

\section{Figure 1}

Models of pluripotency-associated transcription factor network. LIF and Wnt signals are integrated into mouse ES cells to direct the transcriptional activation of pluripotency-associated transcription factors Tbx3, Klf4, Tfcp211 and Esrrb. These transcription factors then enhance the auto-regulation of the core transcription factors Oct3/4 and Sox 2 either directly or indirectly via Nanog. Nanog also works as a positive feedback loop to amplify LIF signal.

\section{Figure 2}

Schematic representation of the molecular mechanisms involved in modulation of the activity of tissue-specific transcription factors. Tissue-specific transcription factors mainly target the distal enhancer to direct specific gene expression patterns. Multiple transcription factors cooperate to activate the super-enhancer via interaction with the mediator complex. The mediator complex fascilitate the recruitment of the general transcription factors including TFIID on the promoter, which promotes transcription by RNA polymerase II complex (polII). The remodeling complex and epigenetic factors modulate the recruitment of both tissue-specific and general transcription factors on the target chromatin. RNA export and nuclear import of transcription factors also work as regulators of transcription factor function. 


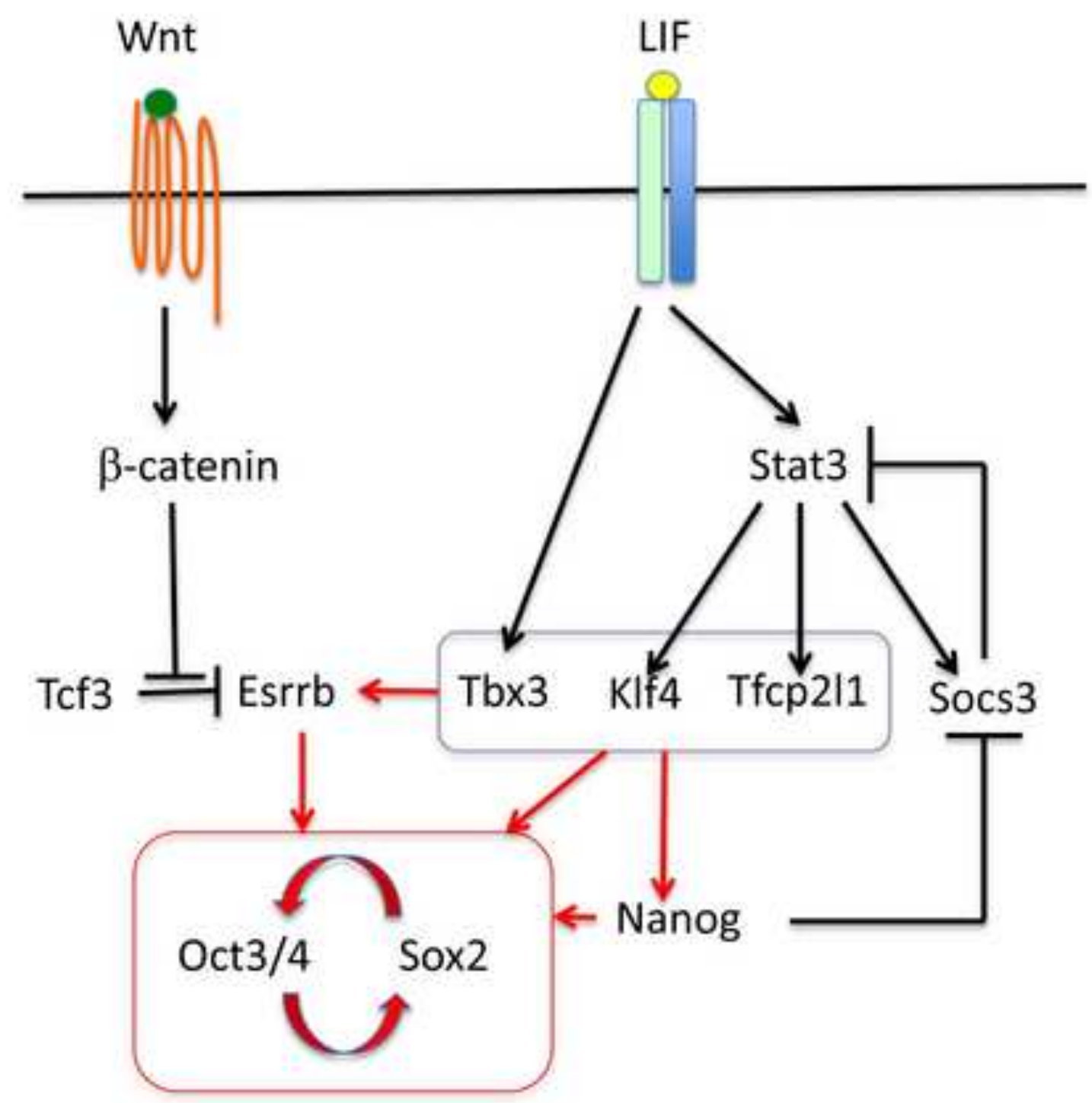




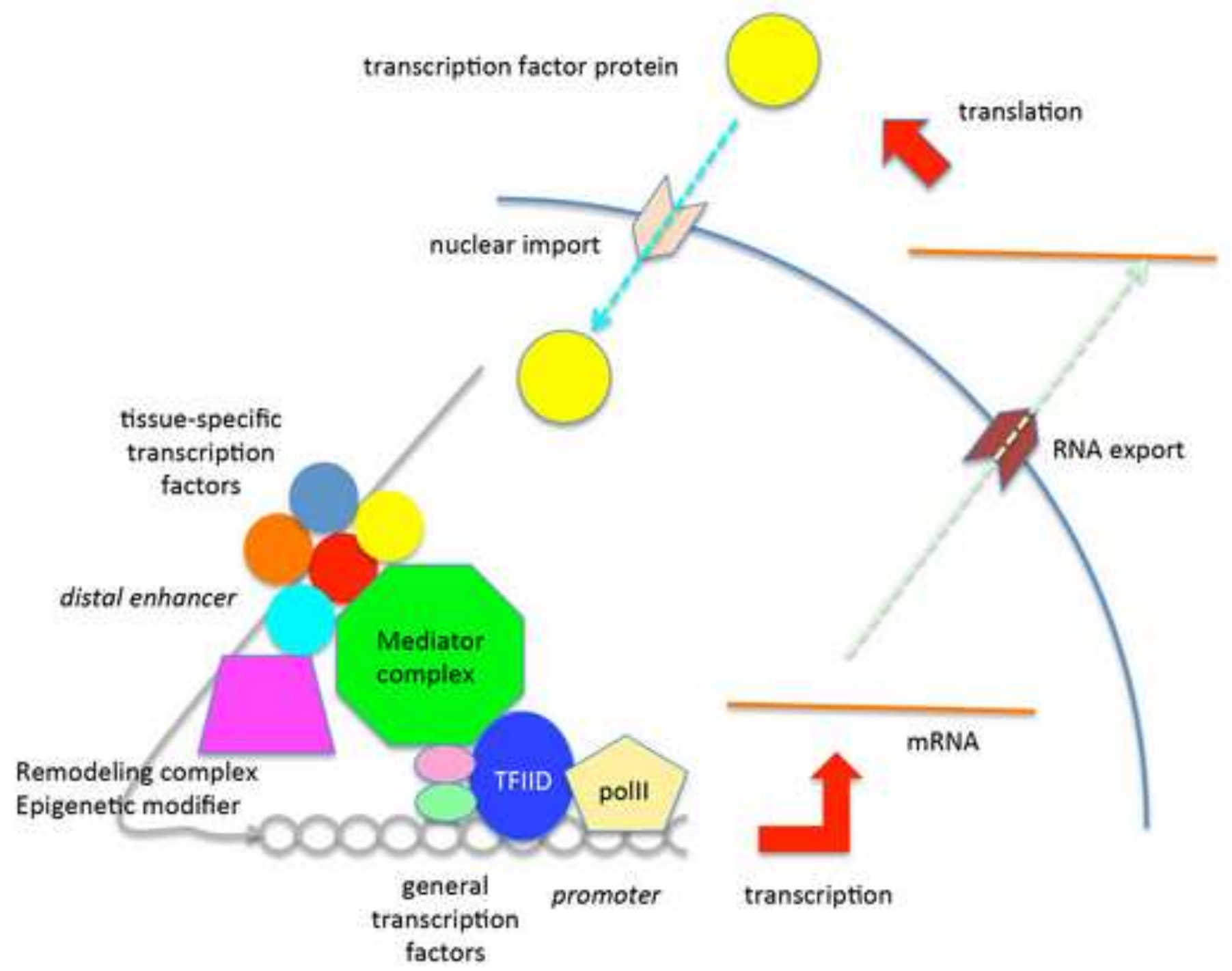

